\title{
Ambiguidade, Negação e C-Comando ${ }^{1}$
}

\author{
Ambiguity, Negation, And C-Command
}

Rerisson Cavalcante de Araújo*

Resumo: Neste artigo, analiso o fenômeno da ambiguidade de escopo negativo em sentenças com adjuntos causais, temporais e de finalidade, em que a negação pode recair sobre o predicado ou sobre o adjunto. Discuto as análises de Huang (1982), Takubo (1985) e Johnston (1994), segundo os quais a ambiguidade decorre de diferentes relações de c-comando entre a negação e o adjunto, em decorrência da opcionalidade de adjunção ao VP e ao IP. Apresento uma série de fatos linguísticos problemáticos para essa análise, i.e., contextos em que o adjunto deve ser c-comandado pela negação, mas em que a ambiguidade ainda permanece, ao contrário do esperado. Argumento que o c-comando é uma condição necessária, mas não suficiente, para a definição do escopo e defendo que a disponibilidade das leituras de negação de predicado e de negação de adjunto decorre da opcionalidade da aplicação de rotulação no processo de adjunção, segundo Hornstein e Nunes (2008).

Palavras-chave: Escopo negativo. Adjuntos. Rotulação.

Abstract: In this paper, I analyze negative scope ambiguity in sentences with cause, temporal and purpose adjuncts, in which negation can take scope either over the predicate or over the adjunct. I discuss Huang (1982), Takubo

\footnotetext{
* Graduado em Letras Vernáculas pela Universidade Federal da Bahia. Mestre em Letras pela UFBA. Doutor em Linguística pela USP. Atualmente é professor de Linguística pela UFBA. Contato: rerissonaraujo@yahoo.com.br.
}

1 Agradeço a Jairo Nunes, Norbert Hornstein, Marcelo Ferreira, Esmeralda Negrão e Mary Kato por comentários a versões anteriores deste trabalho. A pesquisa foi financiada pela FAPESP (2008/00073-0) e pela CAPES (0306-10-0). 
(1985) and Johnston's (1994) analyses, which propose that the ambiguity is derived from different c-command relations between negation and adjunct, due to an optionality of adjunction to VP or IP. Against this view, I show a set of facts which are problematic for any analysis based only in c-command, i.e., several syntactic contexts where negation should c-command the adjunct, but where the ambiguity surprisingly still holds. Based on this, I argue that c-command is a necessary, but not sufficient condition to establish negative scope. And I suggest that the availability of predicate negation and adjunct negation results from an optionality of the operation of Labeling in adjunction, according Hornstein and Nunes (2008).

Keywords: Negative scope. Adjunction. Labeling.

\section{Introdução}

Uma sentença como (1a), com um marcador negativo pré-verbal (ou pré- $\mathrm{I}^{\circ}$ ) e um adjunto causal, é ambígua. Em uma interpretação, a negação recai apenas sobre o evento expresso pelo predicado, excluindo o adjunto (N-Pred), como mostra (1b); (ii) em outra, a negação recai sobre o adjunto (N-Adj), excluindo o predicado, como mostra (1c).

(1) a. Maria num² foi promovida porque é negra.

b. 'Maria não foi promovida e o motivo disso foi ser negra'. (N-Pred)

c. 'Não foi por ser negra que Maria foi promovida'. (N-Adj)

A mesma ambiguidade ocorre com adjuntos temporais e finais, como em (2) e (3).

(2) a. O Banco Central num baixou os juros para combater a inflação. b. 'O BC não baixou os juros e o objetivo foi combater a inflação' (N-Pred)

\footnotetext{
2 Nos meus exemplos, usarei "num" para o marcador pré-verbal e "não" para o pós-verbal.
} 
c. 'O BC baixou os juros, mas o objetivo não foi combater a inflação' (N-Adj)

(3) a. Ele num saiu depois da meia-noite.

b. 'Depois da meia-noite, ele não saiu (mais)'.

c. 'Ele saiu, mas não (foi) depois da meia-noite'.

(N-Pred)

(N-Adj)

Essa ambiguidade também ocorre em outras línguas. Lasnik (1972) aponta que, em inglês, sentenças como (4) têm as interpretações indicadas em $(4 b-c)$.

(4) a. George doesn't beat his wife because he loves her.

G. AUX-NEG bater sua esposa porque ele ama ela

b. 'George não bate na esposa e o motivo disso é porque ele a ama' (N-Pred)

c. 'George bate na esposa, mas não porque a ama' (N-Adj)

A leitura de N-Pred está associada à existência de duas curvas prosódicas distintas: uma envolvendo o predicado; outra, o adjunto. Já $\mathrm{N}$-Adj está associada a uma curva prosódica única, descendente, abarcando predicado e adjunto. Trata-se, portanto, de um fenômeno que conjuga semântica, sintaxe e prosódia.

Neste artigo, discutirei análises existentes na literatura gerativista que tentam explicar o fenômeno como resultado de uma ambiguidade nas relações sintáticas entre os constituintes, mais especificamente, devido a diferenças nas relações de c-comando entre a negação e o adjunto (seção 1). Embora compartilhe do pressuposto de que a estrutura sintática seja a responsável por produzir esses efeitos semânticos e prosódicos, apresento uma série de fatos problemáticos para a análise baseada exclusivamente em diferenças de c-comando: contextos em que, segundo a análise, uma das leituras deveria ser excluída, mas em que a ambiguidade permanece (seção 2). Por fim, apresento uma análise alternativa baseada na opcionalidade da aplicação da operação de rotulação aos adjuntos, conforme Hornstein e Nunes (2008) (seção 3 e conclusão). 


\section{Análises Prévias}

\subsection{Lasnik (1972)}

Analisando o fenômeno mais geral da interação entre a negação e elementos quantificadores, Lasnik (1972) apresenta duas análises para dar conta das possibilidades de escopo negativo.

Pela Determiner Theory (DT), a negação seria um modificador ou determinante, na Estrutura Profunda (DS), da categoria sobre a qual tem escopo. Assim, o marcador pode ser gerado (i) diretamente sob a categoria Aux(iliar), gerando N-Pred; (ii) ou como modificador de certos advérbios e quantificadores, produzindo N-Adj. No segundo caso, a negação seria posteriormente movida para Aux por uma transformação Not Shift, derivando uma sentença que corresponde, superficialmente, a uma negação sentencial, mas semanticamente a uma negação de constituinte.

Por esta análise, uma sentença como (5a) poderia ser gerada a partir de duas DSs distintas. A leitura de N-Adj seria derivada da DS em (6a), com a negação gerada como modificador do adjunto (posteriormente movida para Aux). Já N-Pred seria derivada da DS em (6b), em que a negação é gerada diretamente sob Aux. A ambiguidade da sentença (4a), repetida em (7a), seria derivada das estruturas em (7b) e (7c).

(5) a. I don't attend class often.

b. 'Frequentemente, eu não assisto as aulas' (N-Pred)

c. 'Eu assisto as aulas, mas não frequentemente' (N-Adj)

(6) a. DS de N-Adj

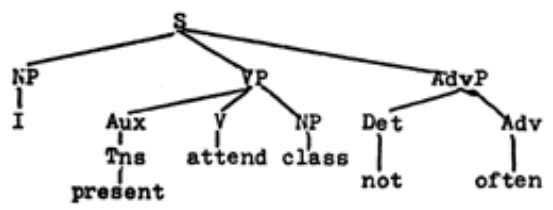


b. DS de N-Pred

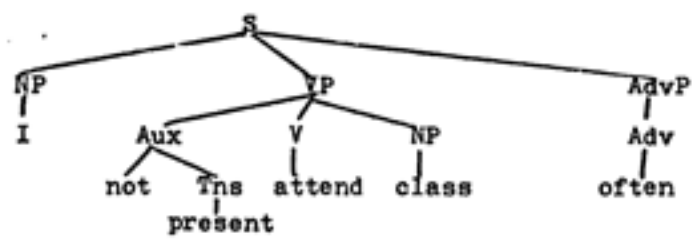

(LASNIK, 1972, p. 60)

(7) a. George doesn't beat his wife because he loves her.

DS de N-Pred:

b. $\left[_{\mathrm{S}}\left[_{\mathrm{NP}}\right.\right.$ George $]\left[_{\mathrm{VP}}\right.$ not Aux beat his wife $]\left[_{\mathrm{AdvP}}\right.$ because... $\left.\left.\left.]\right]\right]\right]$

DS de N-Adj:

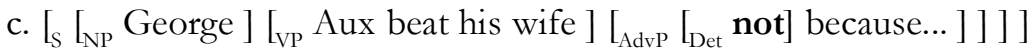

O maior problema da DT é o quadro teórico em que foi desenvolvida, incompatível com várias pressuposições atuais da teoria gramatical. Mais problemático do que o apelo a estruturas profundas e superficiais é o pressuposto de que a interpretação semântica seja definida (exclusivamente) pela DS, não sendo afetada pelas transformações e movimentos subsequentes.

O próprio Lasnik (1972) aponta que essa análise não tem como relacionar as duas interpretações às diferenças de entonação, uma vez que as regras prosódicas só se aplicam após a transformação Not Shift, quando as estruturas são idênticas e não há como PF (Phonetic Form) identificarem diferenças entre as duas leituras.

A segunda análise de Lasnik, a Pre S Theory, é proposta especificamente para sentenças como (8), em que a negação seria gerada em posição pré-sentencial $(=\mathrm{CP})$ e haveria um fronteamento do adjunto para uma posição entre a negação e o sujeito (além da aplicação de regras de reajuste), gerando N-Adj. 
(8) a. Not often does John pass a test.

(LASNIK, 1972, p. 33)

b. DS

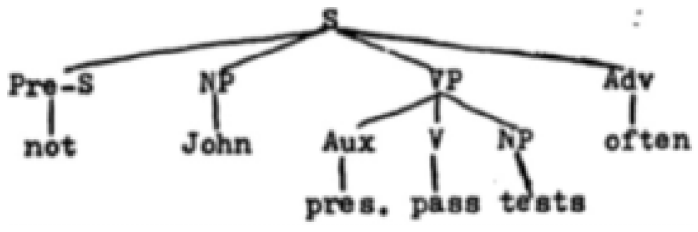

c. SS

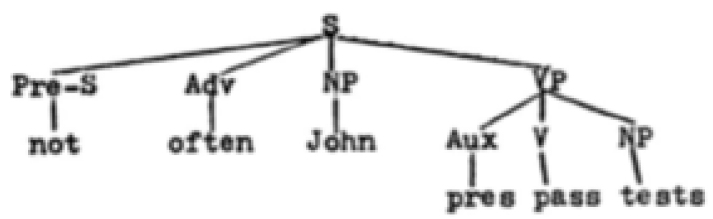

Essa análise não pode ser aplicada aos casos de (5) e (7), em que não há fronteamento do adjunto e em que há ambiguidade. Para tais casos, Lasnik (1972) propõe uma solução baseada em uma regra prosódica. As leituras de N-Pred e de N-Adj teriam a mesma estrutura sintática. A ambiguidade não seria resultado de derivações distintas, mas de uma regra prosódica que atribuiria à sentença contornos entonacionais diferentes, resultando em diferentes interpretações semânticas. Ou seja, uma regra fonológica com repercussões semânticas, que teria, segundo o autor, um efeito semelhante ao processo de topicalização.

A postulação de uma regra pós-sintática que relaciona a entonação à interpretação semântica, sem a mediação da sintaxe, parece mais uma descrição do resultado com que nos deparamos do que uma explicação para o comportamento dessas sentenças. O próprio Lasnik (1972) reconhece o status problemático de uma regra de tal tipo no modelo gerativo, em que interpretação fonética e interpretação semântica não possuem ligação direta, mas são mediadas pela estrutura sintática.

É plausível especular que a interpretação semântica não é resultado de uma regra de entonação indiferente à sintaxe, mas que, pelo contrário, a prosódia e a semântica são resultado de diferenças na estrutura sintática das 
sentenças. Essa é a intuição em que se baseiam as análises de Huang (1982), Takubo (1985) e Johnston (1994), que discuto a seguir.

\subsection{Huang (1982) e Takubo (1985)}

Huang (1982), também trabalhando com a interação da negação com quantificadores, discute as diferenças de escopo negativo com adjuntos "motivacionais" (causa, finalidade) e temporais a partir de dados do inglês e do chinês. O autor propõe que a diferença de escopo resulta das relações de c-comando.

Essa diferença seria mais transparente no caso do chinês, em que há alteração na ordem linear em função da interpretação licenciada. A sentença (9a) tem a leitura de N-Adj; e (9b), de N-Pred.

(9) Chinês (HUANG, 1982, p. 111-112)

a. Zhangsan meiyou [ yinwei tá pioliang] jiehum. (N-Adj)

not because she pretty marry

('Z. se casou, não porque ela fosse bonita')

b. Zhangsan [ yinwei ta pioliang ] meiyou jiehum. (N-Pred) because she pretty not marry ('Zhangsan não se casou e o motivo foi ela ser linda')

Esses dados, na verdade, mostram que não há ambiguidade de escopo com esse tipo de adjunto em chinês, pois cada leitura apresenta uma ordem linear distinta. Mais importante, a leitura de N-Adj só ocorre quando a negação precede o adjunto, sugerindo que a ordem linear é a responsável por determinar o escopo. Huang (1982), entretanto, adota uma análise baseada, não na ordem linear, mas na estrutura sintática. $O$ autor propõe que as diferenças de escopo em (9) decorrem de diferentes relações de c-comando ${ }^{3}$,

3 Tradicionalmente, $\alpha$ c-comanda $\beta$ se e somente se:

(i) $\alpha$ não domina $\beta$;

(ii) $\beta$ não domina $\alpha$;

(iii) o primeiro nó ramificante que domina $\alpha$ também domina $\beta$. 
que também se refletem na ordem dos constituintes. Em (9a), a negação c-comanda o adjunto, gerando N-Adj. Em (9b), o adjunto estaria em uma posição hierarquicamente mais alta do que a negação e, por isso, não cairia sob o seu escopo.

Isso decorreria da possibilidade de o adjunto conectar-se a diferentes camadas do VP. Os casos de ambiguidade do inglês, como em (10a), com o adjunto à direita do VP, são explicados, por essa proposta, assumindo que os adjuntos podem estar conectados à camada mais baixa do VP (abaixo de didn't), como em (10c), ou à camada mais alta do VP (que contém didn't), como em (10b).

(10) a. John didn't show up always.

b. John [ [ didn't [ show up ] ] always ] (N-Pred)

'Sempre, John não aparece'

c. John [ didn't [ [ show up ] always ] ] (N-Adj)

'Nem sempre John aparece'

(HUANG, 1982, p. 145)

A análise também consegue dar conta da ausência de ambiguidade quando o adjunto está à esquerda da negação, em inglês. O adjunto está adjungido a uma categoria mais alta do que a negação e não seria c-comandado por ela.

(11) a. [ Always [John [ didn't [ show up ] ] ] (N-Pred)

b. [ John [ always [ didn't [ show up ] ] ] ] (N-Pred)

(HUANG, 1982, p. 145)

Uma análise na mesma linha é explorada por Takubo (1985) para as diferenças entre o japonês e o inglês. A comparação entre as duas línguas é importante para esta discussão, pois a principal diferença entre elas é que o escopo do marcador not é para a direita, enquanto, em japonês, o escopo de nai é para a esquerda, o que fornece um modo de distinguir entre o papel da ordem linear e o do c-comando. Takubo (1985) aponta que o escopo de not 
pode se estender até o final da sentença, mas o escopo de nai, aparentemente, não pode. Enquanto sentenças inglesas como (12) são ambíguas, as sentenças japonesas, em (13), têm apenas a leitura de N-Pred. O adjunto não cai sob o escopo de nai.

(12) I did not buy watches in Paris.

'Em Paris, eu não comprei relógios' (N-Pred)

'Não foi em Paris que eu comprei relógios' (N-Adj)

(TAKUBO, 1985, p. 38)
a. Watasi-wa Pari-de tokei-o kawa-na-katta. (N-Pred) I-TOP in-Paris watches did not buy
( = 'Em Paris, eu não comprei relógios')
b. Watasi-wa 1920-nen-ni-wa umare-te-i-na-katta.
(N-Pred)

I-TOP in- 1920-TOP had-not-been-born
( = 'Até 1920, eu não tinha nascido (ainda)')

(TAKUBO, 1985, p. 36)

Takubo (1985) posiciona-se contra a análise de Kuno (1980), segundo a qual esses fenômenos seriam resultado de o marcador negativo do japonês só poder ter escopo sobre o elemento verbal que o precede imediatamente, não podendo ter escopo além deste elemento. Takubo (1985) aponta que esta análise, além de não explicar a motivação para essa diferença nas duas línguas, é empiricamente incorreta, já que há casos em que o escopo negativo de nai pode se estender para além do verbo que o precede. É o caso de sentenças como (14a), em que a negação afixada ao verbo principal pode ter escopo sobre a sentença encaixada, resultando em uma interpretação de Neg-raising, assim como a da sentença equivalente em inglês (14b).

a. Watasi-wa [ kare-ga 1920-nen-ni umareta to ] (wa) omowa-nai. I-TOP he-NOM in-1920 was-born COMPL

(TOP) think-NEG

'Não acho que ele nasceu em 1920'. / 'Acho que ele não nasceu em 1920'. 
b. I don't think he was born in 1920 .

'Não acho que ele nasceu em 1920'. / 'Acho que ele não nasceu em 1920'.

A leitura de N-Adj também se torna disponível se as sentenças forem nominalizadas (ou clivadas) (cf. (15)).

(15) a. Watasi-wa Pari-de tokei- o katta no dewa nai. (N-Adj)

I-TOP in-Paris watches buy COMPL COP NEG

('Comprei relógios, (mas) não em Paris'.)

b. Kanozyo-ga kanemoti-datta kara, kanozyo-to kekkon-sita no dewa nai.

she-NOM was rich because with-her marry COMPL COP NEG

('Casei, não porque ela era rica')

(TAKUBO, 1985, p. 40)

Takubo (1985), então, adota a análise baseada em Huang (1982) de que o domínio da negação é por c-comando. Para o inglês, assume, junto com Huang (1982), que o adjunto pode ser um constituinte de VP ou da sentença, gerando as leituras de N-Adj ou N-Pred, respectivamente. Para o japonês, ele postula que esta é uma língua não configuracional, que não possui VP. As sentenças em (13) teriam estruturas semelhantes à representada em (16), com nai afixado ao verbo, mas com o adjunto e o complemento como constituintes imediatos de S, fora do c-comando e do escopo de nai.

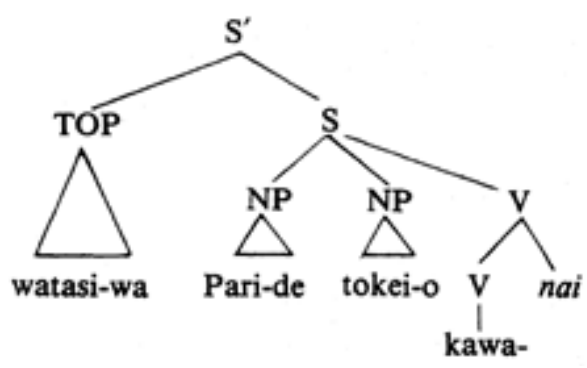

(TAKUBO, 1985, p. 41) 
A hipótese da ausência de VP não é compatível com as pressuposições atuais da teoria sintática, mas a representação em (16) pode ser traduzida em termos de movimento ou scrambling do adjunto e do complemento para uma posição acima do VP e da negação.

Para os casos de (14)-(15), em que a negação tem escopo além do verbo a que está afixada (gerando N-Adj), Takubo (1985) propõe que os constituintes introduzidos pelo complementador no formam um constituinte com o verbo, caindo, portanto, sob o c-comando e escopo da negação nai. Novamente, não é necessário assumir que o japonês seja não configuracional nem que as sentenças com o complementizador no constituam algum tipo de exceção. Assumindo que o japonês é uma língua com núcleo final, a análise pode ser traduzida para uma versão configuracional segundo a qual as completivas em (15) são complemento do verbo e estão, portanto, sob o c-comando da negação, enquanto os adjuntos em (13) estão deslocados para uma posição mais alta, possivelmente por scrambling.

As análises de Huang (1982) e Takubo (1985) conseguem, portanto, dar conta dos dados, mas apresentam um problema. Se os adjuntos causais podem ser gerados em diferentes posições da estrutura, segue-se facilmente a impossibilidade de N-Adj quando estes são gerados acima da negação. Também está claro por que a leitura de N-Adj passa a estar disponível quando os adjuntos são gerados abaixo da negação, mas não é claro por que (9a) e (10c) permitem apenas a leitura de N-Adj, pois, nestes casos, o predicado continua sendo c-comandado pela negação, ainda que o adjunto também passe a sê-lo. A previsão é de que essas estruturas resultassem em uma negação simultânea do predicado e do adjunto ${ }^{4}$.

Uma pista sobre como explicar por que a posição baixa do adjunto bloquearia a leitura de N-Pred vem do trabalho de Johnston (1994), que discuto a seguir.

${ }^{4}$ Um problema adicional é a compatibilidade da análise de Huang (1982) e Takubo (1985) com o Axioma de Correspondência Linear de Kayne (1994), segundo o qual c-comando assimétrico implica precedência linear. Isto faz a previsão de que o adjunto em IP deveria preceder a negação, ao contrário do que ocorre. Não discutirei esse problema aqui. Apenas considerarei plausível que o mecanismo de determinação da ordem linear funcione de modo diferente para os adjuntos (cf. CHOMSKY, 1995). 


\subsection{C-comando e adjunção ao VP ou ao TP}

Outra formulação da hipótese da ambiguidade nas relações de c-comando é encontrada no trabalho de Johnston (1994) sobre o escopo negativo sobre adjuntos e o licenciamento de itens de polaridade negativa em inglês. O autor defende a mesma ideia de Huang (1982), de que as leituras de N-Pred e de N-Adj são derivadas da possibilidade de o adjunto causal ser gerado em posições distintas na estrutura sentencial.

Johnston (1994) baseia essa hipótese na ideia de que uma preposição como because tem a função de estabelecer uma relação entre proposições ${ }^{5}$ e, por isso, pode se adjungir a qualquer categoria que denote uma proposição. Considerando que o sujeito é gerado internamente ao VP, Johnston (1994) assume que, além do IP/TP, o VP também corresponde a uma proposição; e conclui que as because sentences podem se adjungir não apenas ao IP/TP, mas também ao $\mathrm{VP}^{6}$. Adjungidas ao VP (cf. (17b)), elas ficariam sob c-comando da negação, alojada em I ${ }^{\circ}$, gerando a leitura de N-Adj. Adjungidas ao IP (cf. (17c)), elas ficariam acima da negação e fora de seu escopo, gerando a leitura de N-Pred.

(17) a. Marty didn't sell his bike because the gears were broken.

b. N-Adj: 'Marty vendeu sua bicicleta, (mas) não porque a marcha estava quebrada'

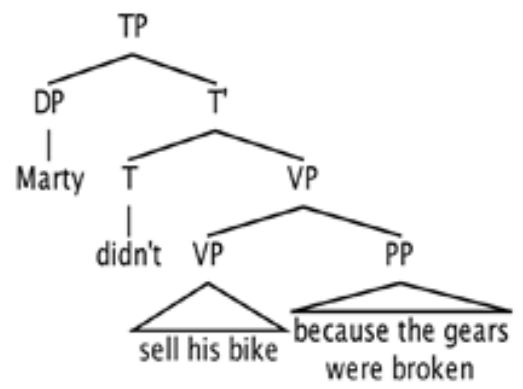

${ }^{5}$ Note, porém, o leitor que essa generalização não é correta:

i) He didn't come because of you.

${ }^{6}$ Note-se que, para Johnston, a adjunção ao IP/TP é que é tomada como certa, e a adjunção ao VP é que precisa ser provada/proposta. 
c. N-Pred: 'Marty não vendeu sua bicicleta e o motivo é porque a marcha estava quebrada'

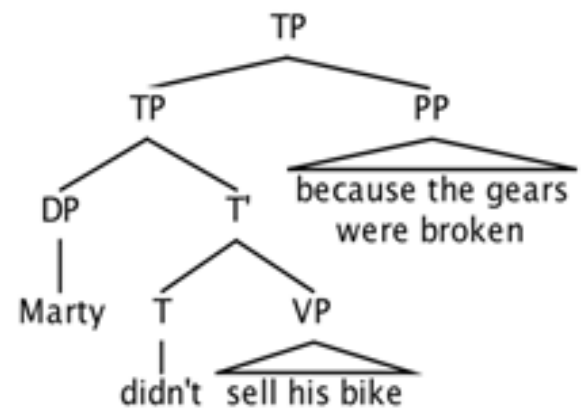

(JOHNSTON, 1994)

Johnston (1994) apresenta três argumentos empíricos em favor da opcionalmente de adjunção a IP ou a $\mathrm{VP}^{7}$. Primeiro, quando esse tipo de adjunto ocorre à esquerda da sentença matriz, como em (18), apenas N-Pred é permitida, o que é esperado se assumirmos que, nesta posição, a adjunção é ao IP, não ao VP.

(18) Because the gears were broken, Marty didn't sell his bike. (N-Pred) 'Porque a marcha estava quebrada, Marty não vendeu sua bicicleta'

(JOHNSTON, 1994)

Em segundo lugar, em elipse de VP, o adjunto pode ser ou elidido junto com o VP ou preservado da elipse. Johnston (1994) defende que esse comportamento pode ser explicado se assumirmos que, em (19a), o adjunto do segundo conjunto está no VP e é elidido junto com ele, mas que, em (19b), o adjunto está acima do VP (i.e. no IP) e, por isso, escapa do processo de elipse.

\footnotetext{
${ }^{7}$ O autor, na verdade, considera a adjunção ao IP como o caso básico e tenta provar a possibilidade de adjunção ao VP.
} 
(19) a. If Marty sold his shares because the Market was unstable, then Leopold did.

'Se Marty vendeu suas ações porque o mercado estava instável, então Leonard (também) vendeu'.

b. If Marty sold his shares because the market was unstable, then Leopold did because the profits were high.

'Se Marty vendeu suas ações porque o mercado estava instável, então Leonard vendeu porque os lucros estavam elevados'

(JOHNSTON, 1994)

Em terceiro lugar, há o efeito que a adição de uma tag question tem sobre uma sentença negativa com um adjunto causal. $\mathrm{O}$ autor aponta que uma tag question em uma sentença como (20) força a leitura de N-Adj. Isso é esperado se as tag questions são formadas por uma operação de elipse de VP e se assumirmos que as because sentences podem se adjungir ao VP ou a IP, com a leitura de N-Adj resultando da adjunção ao VP.

(20) a. Marty didn't sell his bike because the gears were broken, did he? (N-Adj)

b. Marty didn't [VP sell his bike because the gears were broken], did he [सP -sell his bike because the gears were broken]?

Estes três argumentos apresentados por Johnston (1994) fornecem uma base empírica mais sólida para corroborar a hipótese de que os adjuntos causais podem estar adjungidos a diferentes posições na estrutura, apesar da ordem linear idêntica.

A análise de Johnston (1994) também fornece uma pista sobre como lidar com a exclusão de N-Pred quando o adjunto está abaixo da negação. Baseando-se em Linebarger (1987), ele aponta que a presença de um NPI no predicado anula a possibilidade de leitura de N-Adj (cf. (21)). Os NPIs a red cent e anymore no predicado principal forçam a leitura de N-Pred. Isso se seguiria do fato de que o escopo da negação sobre o predicado (e, portanto, não apenas o c-comando) é necessário para o licenciamento dos NPIs. 
(21) a. Leopold didn't earn a red cent because the market was unstable.

(N-Pred)

b. Leopold didn't sell shares anymore because the market was unstable.

(N-Pred)

A exclusão de N-Adj é confirmada pela agramaticalidade causada pela adição de uma tag question, para forçar o escopo sobre o adjunto.

(22) a. * Leopold didn't earn a red cent because the market was unstable, did he?

b. * Leopold didn't sell shares anymore because the market was unstable, did he?

Nessa perspectiva, apenas o c-comando da negação sobre o NPI não seria suficiente para o licenciamento. É preciso que o escopo negativo aja especificamente sobre a parte da sentença em que o NPI aparece (i.e., o predicado). A questão que se coloca diante de (21) e (22) é: por que os NPIs no predicado não podem ser licenciados na interpretação de N-Adj uma vez que, mesmo nesta leitura, há uma configuração em que a negação permanece c-comandando os NPIs?

A solução de Linebarger (1987) tem duas partes. Ela propõe que elementos como because, a própria negação e certos advérbios são operadores que precisam se mover em LF para tomar escopo sobre a sentença. Adicionalmente, ela formula uma Restrição de Escopo Imediato (Immediate Scope Constraint), segundo a qual um NPI é aceitável apenas se, em LF, estiver sob o escopo imediato do operador negativo, ou seja, se (i) ocorre em uma proposição que constitui o "escopo completo" da negação e (ii) nessa proposição não há nenhum elemento lógico intervindo entre o NPI e a negação. Em outras palavras, a presença de qualquer operador lógico entre a negação e o NPI bloquearia o escopo imediato, impedindo o licenciamento.

Johnston (1994) assume a ideia de Ladusaw (1988) de que o escopo da negação é determinado pela sua posição superficial antes de LF e, por isso, não adota o requerimento de Linebarger (1987) de movimento do operador negativo e de because, mas adota a ideia básica desta autora de que operadores lógicos como because podem interferir e bloquear o licenciamento 
de NPIs. A adjunção ao VP, abaixo da negação (mas acima dos NPIs), teria o efeito de impedir o licenciamento dos NPIs por parte do marcador negativo em I ${ }^{\circ}$.

Tanto a análise de Linebarger (1987) quanto a implementação de Johnston (1994) tratam da interferência, por parte do adjunto causal, no licenciamento de NPIs a partir de uma falha no escopo imediato, e não do bloqueio do escopo negativo em geral. Ainda assim, uma vez que se assume que o operador causal é capaz de bloquear um (dos) resultado(s) do escopo negativo, pode-se especular que a presença do adjunto poderia também ser responsável pelo efeito mais geral de bloquear a leitura de N-Pred mesmo em sentenças sem NPIs, um resultado contrário ao esperado.

\section{Problemas para as Análises de (Ausência de) C-Comando}

Nas próximas subseções, apresento fatos que são problemáticos para a análise de Huang, de Tabuko e de Johnston (doravante, HTJ), que explicam a ambiguidade de escopo apenas por c-comando.

\subsection{Licenciamento de anáforas em adjuntos}

Um primeiro problema para a análise de HTJ vem do licenciamento de anáforas em adjuntos. Se há diferenças nas relações de c-comando entre $\mathrm{N}$-Pred e N-Adj, isso deveria afetar o licenciamento de uma anáfora interna ao adjunto, mas ligada ao sujeito sentencial.

Os exemplos em (23), porém, mostram que uma anáfora como each other, quando interna ao um adjunto causal, é licenciada nas duas interpretações, contrariando a previsão feita pela análise. (23a) tem a leitura de $\mathrm{N}-\mathrm{Adj}$, com a interpretação de que o respeito mútuo não foi a motivação para os sujeitos praticarem determinada ação. Já (23b) tem a leitura de N-Pred e a interpretação de que, por respeito mútuo, os sujeitos não fizeram (ou desistiram de fazer) algo. 


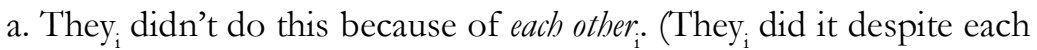
other.)

'Eles não fizeram isso por causa um do outro. Eles fizeram isso apesar um do outro'

\section{N-Pred}

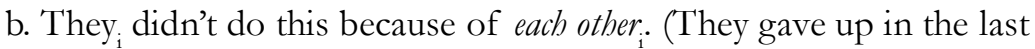
minute.)

'Eles não fizeram isso por causa um do outro. Eles desistiram no último minuto'

Nas duas sentenças, a anáfora é licenciada como correferente ao sujeito e, portanto, deve ser c-comandada por este. Se (23a) e (23b) são aceitáveis, o sujeito sentencial deve c-comandar o adjunto causal tanto em N-Pred quanto em N-Adj. Isso ocorre sem problemas se o adjunto causal estiver adjungido ao VP. Se, porém, a adjunção for ao IP, a maioria das definições de c-comando (em termos de irmandade e/ou de dominância imediata) prevê que não há c-comando por parte do sujeito (em Spec,IP) sobre o adjunto. Estando a negação em $\mathrm{I}^{\circ}$, o resultado é que nem negação nem sujeito c-comandam o adjunto. A análise de HTJ faz a previsão incorreta de que, em N-Pred, o licenciamento de anáforas internas ao adjunto deveria ser bloqueado.

Para contornar esse problema, a análise precisaria assumir que, em N-Pred, o adjunto é gerado em uma posição em que não é c-comandado pela negação, mas é c-comandado pelo sujeito. Podemos especular três tipos de soluções. A primeira seria adotar um conceito de c-comando segundo o qual o especificador de um XP c-comanda um adjunto do mesmo XP. Uma noção tal de c-comando parece ser necessária para explicar o licenciamento de anáforas ou NPIs em adjuntos de $\mathrm{VP}(=\nu \mathrm{P})$, por parte de objetos diretos através do movimento do objeto para o especificador de $v \mathrm{P}$, como em $(24)^{8}$.

${ }^{8}$ Cf. Lasnik e Saito (1991) a respeito. 
Uma definição de c-comando que assume isso está presente no sistema de Kayne (1994), que ignora a distinção entre segmentos e categorias e calcula o c-comando com base apenas na dominância pela primeira categoria. Por essa definição, o especificador e um adjunto se c-comandam mutuamente, o que poderia gerar o resultado necessário de que um adjunto gerado no IP escapa ao c-comando por parte da negação em $\mathrm{T}^{\mathrm{o}}$, mas cai sob o c-comando do sujeito no especificador de IP. Mas a adoção desse conceito de c-comando de Kayne (1994) é problemática, pois envolve uma série de outros pressupostos, além de fazer previsões que afetam todos os processos da faculdade da linguagem que envolvem o conceito de c-comando. Nesse sistema, por exemplo, os especificadores são reduzidos a casos de adjunção.

A segunda solução seria manter a adjunção ao IP, mas assumir que o sujeito sentencial ocorre em uma posição acima de IP, como no especificador de alguma categoria funcional mais alta que o IP. Essa solução vai contra as análises sobre o sujeito sentencial no inglês, que assumem que há adjacência entre o núcleo funcional (e os verbos auxiliares) e o sujeito sentencial.

A terceira solução é simplesmente assumir que esses adjuntos não se adjungem ao IP, mas ao I', onde escapam ao c-comando por parte da negação $\left(\mathrm{em} \mathrm{I}^{\circ}\right)$, mas caem sob o c-comando do sujeito sentencial no especificador de IP.

Nas próximas subseções, apresentarei problemas adicionais para a análise de HTJ e mostrarei também que a solução de assumir que o adjunto deve ser gerado em I' é igualmente problemática, pois gera previsões incorretas quando um elemento negativo ocorre no especificador de IP ou CP.

\subsection{Linguas em que NegP domina IP/TP}

A análise de HTJ pressupõe que a negação seja gerada diretamente na posição de $\mathrm{I}^{\mathrm{o}} / \mathrm{T}^{\mathrm{o}}$ (ou em alguma posição intermediaria entre $\mathrm{I}^{\mathrm{o}}$ e VP, sendo posteriormente deslocada para, no máximo, $\mathrm{I}^{\circ}$ ), de modo a não c-comandar um adjunto causal que esteja adjungido a TP (ou ao T').

Laka (1990), entretanto, argumenta que as relações de seleção entre os núcleos funcionais não são rígidas, mas sujeitas a parametrização (cf. também 
Ouhalla, 1997). A autora argumenta especificamente em favor da alternância entre as categorias NegP e TP (= IP), com a possibilidade de TP dominar NegP (em inglês, por exemplo) ou de NegP dominar TP (em basco, por exemplo) (cf. também Pollock, 1989). Essa alternância faz a previsão de que, em línguas em que NegP seja gerado acima de TP, o adjunto causal sempre esteja sob c-comando da negação, independentemente do seu local de adjunção, a VP ou a TP (ou a T’), como se pode ver em (25).

(25) a.

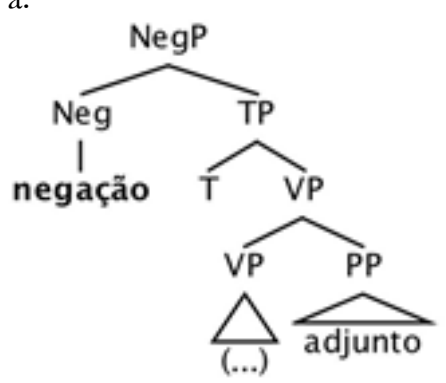

b.

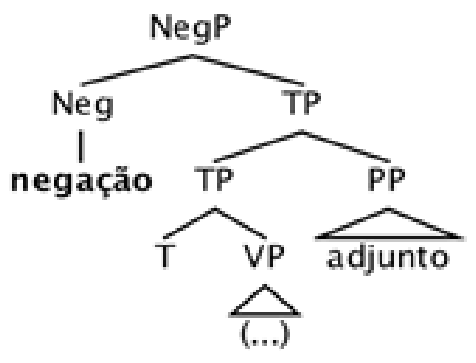

Dadas essas configurações, a previsão é de que uma língua em que NegP domine TP não apresente ambiguidade de escopo negativo, gerando apenas sentenças com a leitura de N-Pred. Isso, entretanto, não se confirma. Segundo Mioto (1992) e Namiuti (2008), o PB é uma língua em que NegP domina todo o IP (formado por AgrP e TP, nesta ordem). Martins (1997) e Fonseca (2004) consideram que NegP é dominado pela categoria mais alta do IP (AgrP no primeiro caso, TP no segundo), mas assumem a existência de uma outra categoria de polaridade sentencial ( $\Sigma \mathrm{P}$ ou PolP, respectivamente) 
acima de AgrP/TP, para a qual a negação e o verbo devem se mover. Como análise proposta especificamente para o PE, temos Martins (1994), que também coloca $\Sigma$ P como gerado acima do T, mas abaixo de AgrP.

Em suma, a hipótese de que, em português, NegP domina TP tem vários defensores na literatura. Apenas na análise de Martins (1994) a negação não c-comandaria um elemento adjungido à categoria flexional mais alta do IP.

Mioto (1992) apresenta os seguintes argumentos em favor dessa configuração no PB (que também servem para o PE): primeiro, gerar NegP acima de TP explica a possibilidade de um quantificador ou advérbio negativo em posição pré-verbal ser licenciado independentemente da ocorrência do marcador negativo pré-verbal, como em (26). O licenciamento ocorreria por uma relação de Spec-núcleo entre o quantificador/advérbio em Spec,NegP e Nego ${ }^{\circ}$ o que dispensaria a manifestação lexical desse núcleo.

Segundo, isso também explica a possibilidade de o mesmo quantificador ou advérbio negativo pré-verbal licenciar itens negativos pós-verbais. Por essa análise, os itens negativos pré-verbais estariam em Spec,NegP, estabelecendo uma relação com o núcleo negativo.

(26) a. Ninguém (*não) comprou nada.

b. Nada (*não) aconteceu.

c. Ele nunca (*não) foi à Bahia.

Terceiro, gerar NegP acima de TP também explica a possibilidade de se elidir o IP, mantendo a negação intacta, ao contrário do que ocorre no inglês, em que é impossível elidir o IP sem apagar a negação junto.

(27) a. Pedro agrediu João, mas Paulo não agrediu Jeão.

(elipse de IP)

b. Pedro não agrediu João e Paulo também não arediu Jeão.

(elipse de IP)

c. Mary bought a book and Peter didn't buy a boek.

(elipse de VP)

d. *Mary has bought a book and Peter has not buy a book.

(elipse de IP) 
Se é correto que NegP domina IP/TP, então uma sentença como (28a) teria as representações em (29a) e (29b), com o adjunto respectivamente no VP ou no TP. Nas duas configurações, o adjunto estaria sob o c-comando da negação e, portanto, (28a) não poderia ser ambígua. Entretanto, a sentença o é.

(28) a. Maria num foi promovida porque é negra.

b. 'Não foi por ser negra que Maria foi promovida'. (N-Adj)

c. 'Maria não foi promovida e o motivo disso foi ser negra'. (N-Pred)

(29) a.

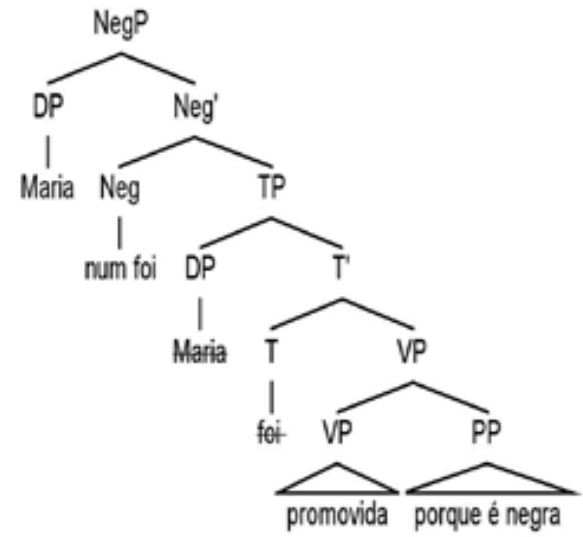

b.

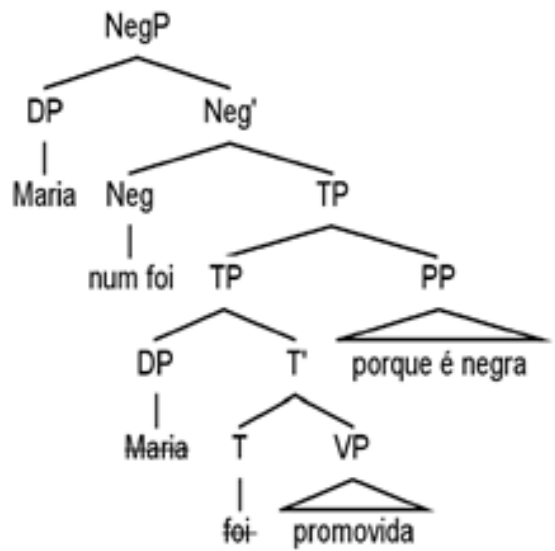


Esses dados mostram que a análise de adjunção ao IP/TP não é suficiente para gerar N-Pred em línguas em que NegP domine o TP, o que permite inclusive colocar em dúvida a validade desta análise para as línguas como TP domine NegP. A quem interessasse salvar a análise baseada em diferenças nas relações de c-comando, haveria duas soluções possíveis. A primeira é questionar a análise de Mioto (1992), A. Martins (1994), E. Martins (1997), Fonseca (2004) e Namiuti (2008), de que NegP domina TP em português. Poderíamos considerar que a ambiguidade de escopo é justamente um argumento contra a análise e, portanto, uma evidência de que, de fato, o português se comporta como o inglês, com TP dominando NegP.

Vitral (1999), por exemplo, adota uma análise distinta, considerando a negação pré-verbal do PB como gerada entre TP e VP. Essa análise ganha força se adotarmos uma concepção de universalidade na hierarquia sentencial, de modo contrário à hipótese de Laka (1990) sobre a parametrização na seleção dos núcleos funcionais.

Uma segunda opção é assumir que NegP, de fato, domina TP em línguas como o português e tentar explicar a persistência da ambiguidade, assumindo que os adjuntos causais podem se adjungir, ao menos nessas línguas, a outras categorias mais altas do que o IP, como o próprio NegP ou o CP.

Nas próximas seções, mostrarei que nenhuma dessas soluções é satisfatória.

\subsection{Sujeitos negativos}

Mesmo excluindo a possibilidade de NegP dominar TP e assumindo que TP sempre domina NegP, outros problemas permanecem. A análise de que TP domina NegP apresenta problemas para as relações de c-comando da negação sobre o adjunto (gerado no T’) quando consideramos dados em que a negação é incorporada no sujeito. A previsão é de que um sujeito negativo, em Spec,TP (acima de NegP), tenha escopo sobre o adjunto, esteja ele no VP quer no T', forçando a leitura de N-Adj e bloqueando N-Pred.

Os exemplos em (30) e (31) mostram que, em português e em inglês, as sentenças com sujeitos negativos permanecem ambíguas. 
(30) a. Ninguém saiu por sua causa.
b. 'Foi por sua causa que ninguém saiu'
(N-Pred)
c. 'Não foi por sua causa que (eles) saíram'
(N-Adj)

(31) a. No student left because of the test.

b. 'Foi por causa do teste que nenhum estudante saiu'.

(N-Pred)

c. 'Não foi por causa do teste que os estudantes saíram'

Além de problemáticos para a análise de HTJ, esses dados mostram que a opção de assumir uma ordem fixa entre TP e NegP não resolve a questão. Mas, antes de descartarmos de vez a análise de HTJ, consideremos ainda outra forma de implementação técnica, a saber, a ideia de que o adjunto pode ser gerado em VP ou em CP (ao invés de em TP), que ganha força, inclusive, em função da visão mais recente da teoria de fases, que considera que $\nu \mathrm{P}$ e CP definem proposições (cf. Chomsky, 2000). Nas próximas subseções, apresento dois tipos de argumentos contra a solução de adjunção ao CP.

\subsection{Controle em adjuntos}

Adotando a hipótese de que os adjuntos causais podem se adjungir ao CP (ou a C'), a leitura de N-Pred seria gerada pela estrutura em (32a), e a de $\mathrm{N}$-Adj por (32b).

a. N-Pred

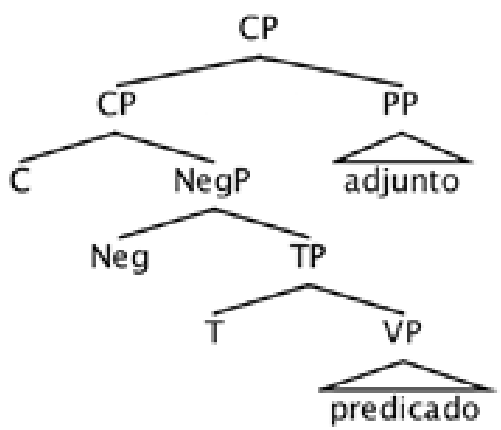


b. N-Adj

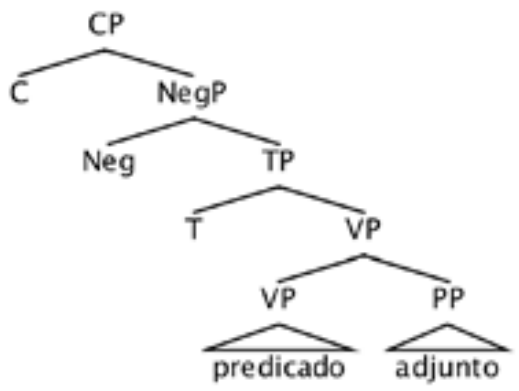

Contudo, a opção de adjunção ao C'/CP já enfrenta um problema com o licenciamento de anáforas (cf. seção 2.1). Uma anáfora interna a um adjunto é licenciada pelo sujeito da matriz, tanto em N-Adj (o que é o esperado por (32b)) quanto em N-Pred, o que é inesperado na configuração em (32a), em que o sujeito não c-comando a adjunto. Por (34a), a anáfora não poderia ser licenciada em N-Pred.

Outro problema para a opção de adjunção ao C'/CP vem das estruturas de controle, por parte do sujeito matriz, em sentenças adjuntas. Sentenças como (33) mostram que o sujeito nulo da sentença infinitiva adjunta é interpretado como correferente ao sujeito da matriz em uma relação de c-comando.

(33) a. I gave Scruffy a biscuit (in order) $\__{1}$ to keep him quiet.

'Eu dei um biscoito a Scruffy para mantê-lo quieto'

b. Mary ${ }_{1}$ escaped, only _ 1 to be recaptured.

'Mary fugiu apenas para ser recapturada'

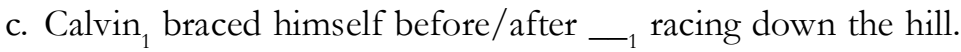
'Calvin preparou-se antes/depois de descer ladeira abaixo'

(URK, 2010, p. 41)

Se, em N-Pred, o adjunto é gerado em CP ou em outra projeção funcional acima daquela que aloja o sujeito, a previsão é de que, nessa leitura, 
o controle falhe por não haver relação de c-comando entre o sujeito matriz e sujeito nulo da sentença infinita adjunta. Essa previsão, entretanto, se mostra incorreta. As versões negativas de (33), em (34), mostram a mesma ambiguidade de escopo. Curiosamente, a única exceção é (33b/34b), em que, por motivos independentes, uma das leituras é praticamente proibida, mas essa leitura é justamente a de N-Pred. A substituição de be recaptured por outra expressão que não seja antônimo de escape, faz com que a sentença seja novamente ambígua (cf. (34c)).

(34) a. I didn't give him a biscuit (in order) _ 1 to keep him quiet. 'Eu dei não um biscoito a ele e o motivo foi para mantê-lo quieto' (N-Pred) 'Eu dei um biscoito a ele, mas o motivo não foi para mantê-lo quieto'

b. Mary ${ }_{1}$ didn't escape only __ to be recaptured. \# 'Mary não fugiu e acabou sendo recapturada' (\#N-Pred) 'Mary fugiu, mas não acabou sendo recapturada' (N-Adj)

c. Mary ${ }_{1}$ didn't escape only __ 1 to see his family. 'Foi apenas para ver sua família que Mary não fugiu' (N-Pred) 'Não foi apenas para ver sua família que Mary fugiu' (N-Adj)

d. Calvin ${ }_{1}$ didn't braced himself before __ racing down the hill. 'Antes de descer ladeira abaixo, Calvin não se preparou' (N-Pred) 'Calvin preparou-se, mas não antes de descer ladeira abaixo' (N-Adj)

\subsection{Outras formas negativas acima de TP}

Ainda que se insista na possibilidade de adjunção ao C' ou CP (ou outra categoria mais alta do que $\mathrm{NegP} / \mathrm{TP}$ ), essa análise encontra outros problemas quando consideramos casos de sentenças com elementos negativos realizados em posições claramente mais altas do que o TP. Há pelo menos dois tipos de dados considerarmos aqui. 
O primeiro é o de elementos negativos no Spec,CP, como nos casos de inversão negativa do inglês, como em (35).

(35) a. Never has Mary got a promotion because she is black.

'Mary nunca ganhou uma promoção e o motivo foi por ela ser negra'

(N-Pred)

'Ser negra nunca foi a causa para Maria ganhar promoções'

(N-Adj)

b. Never has Mary got a promotion because of her father.

'Mary nunca ganhou uma promoção e o motivo foi o seu pai'

(N-Pred)

'O pai de Maria nunca foi a causa para ela ganhar promoções'

(N-Adj)

$\mathrm{O}$ advérbio never no Spec,CP c-comanda o adjunto causal que estiver adjungido a quaisquer categorias mais baixas que o CP, como o VP, o IP ou alguma outra categoria. A adjunção ao C' também faz a previsão de que, nos casos de inversão negativa, a negação sempre terá escopo sobre o adjunto causal e apenas a leitura de negação de adjunto estará disponível. Essa previsão falha novamente. As sentenças em (35) são ambíguas entre N-Adj e N-Pred.

Dados equivalentes ao fenômeno de inversão negativa são difíceis de reproduzir no $\mathrm{PB}$, pela ausência de movimento $\mathrm{I}^{\mathrm{o}}$-para- $\mathrm{C}^{\mathrm{o}}$ e porque muitas análises consideram que o advérbio nunca, quando em posição inicial, está adjungido ao TP ou é um especificador de NegP (cf. Mioto, 1992). Ainda assim, uma sentença como (36a), que soa quase erudita no PB, ainda é percebida como ambígua apesar de não ser produtiva. Também no PE, em que a inversão entre sujeito e auxiliar é mais produtiva, (36a) é ambígua.

(36) a. Nunca teriam os meninos feito isso por sua causa.

b. 'Os meninos nunca fariam isso e a causa disso é você'. (N-Pred)

c. 'Você nunca seria a causa para os meninos fazerem isso'. (N-Adj)

Os casos de inversão negativa excluem a possibilidade de a leitura de N-Pred ser gerada por adjunção ao C'. Quanto à adjunção ao CP, isso 
depende da definição de c-comando e da possibilidade de um especificador ter escopo sobre um adjunto, opção que, em meu ponto de vista, é mais apropriada, por permitir explicar dados como (24).

$\mathrm{O}$ segundo caso de elementos negativos gerados em posições mais altas que IP é a coordenação de orações por conjunções negativas, como no uso de neither e nor, em inglês, em sentenças como (37), que também apresenta ambiguidade entre N-Pred e N-Adj. Qualquer que seja a forma mais adequada de representar as estruturas do tipo [neither sentença nor sentença], parece claro que essas conjunções negativas estão estruturalmente mais altas e, portanto, c-comandam as sentenças que introduzem, inclusive seus adjuntos causais, quer eles estejam em VP, em TP ou em CP. Na análise de HTJ, a previsão é de que tais sentenças deveriam ter apenas a leitura de $\mathrm{N}$-Adj, contrariamente ao que ocorre.

(37) a. Neither did John leave because of you nor did Mary come because of me.

b. Neither John left because of you nor did Mary come because of me.

N-Pred

c. 'Foi por sua causa que John não saiu; e foi por sua minha causa que Mary não veio'

$\mathrm{N}$-Adj

d. 'Não foi por sua causa que John saiu; e não foi por minha causa que Mary veio'

Em suma, embora o c-comando seja necessário para gerar a leitura de N-Pred, há dados suficientes de adjuntos c-comandados pela negação, mas fora de seu escopo, para considerarmos que algum outro fator, distinto do c-comando, é o verdadeiro responsável pela ambiguidade de escopo negativo. Apresento ainda outro caso problemático para HTJ em 2.6.

\subsection{Linguas como marcadores de negação pós-VP}

A ambiguidade de escopo negativo ocorre em línguas em que a negação antecede o verbo finito (português, espanhol, italiano) e naquelas 
em que o marcador é considerado pós-verbal por seguir (imediatamente) o verbo finito (inglês, holandês, alemão). Isto é, em línguas de negação pré-I ${ }^{\circ}$ e pós- $\mathrm{I}^{\mathrm{O}}$ (mas pré-VP), o que mostra que a ambiguidade é independente da posição do marcador em relação ao verbo finito.

Porém, línguas em que ocorre um segundo marcador negativo, não em posição pós-verbal, mas especificamente pós-VP, manifestam um comportamento diferente. No $\mathrm{PB}$, no crioulo palenquero e no crioulo sãotomense, por exemplo, além do marcador negativo pré- ${ }^{\circ}$, um segundo marcador pode ocorrer em posição final de sentença, seguindo complementos e adjuntos (cf. (38), (39) e (40)).

(38) Nu krelo nu.

NEG crer NEG (= 'Não creio')

(Palenquero; DIECK, 2000, p. 52)

(39) E na se piska fa.

(São-tomense; HAGEMEIJER, 2007, p. 174) 3sG NEG saber peixe NEG (= 'Ele não sabe pescar')

Porém com adjuntos causais, finais e temporais, o segundo marcador tem a opção de antecedê-los ou segui-los. A presença do marcador pós-VP tem o efeito de retirar a ambiguidade da sentença, como em (40), (41) e (42), que não são ambíguos. Curiosamente, a posição do segundo marcador determina o escopo de modo oposto ao previsto: o escopo negativo não recai sobre o elemento que segue a negação, mas sobre aquele que a antecede.

(40) a. Maria num foi promovida não, porque é negra.

(N-Pred)

b. Maria num foi promovida porque é negra não.

(N-Adj)

(41) a. Ele num voltou pra casa não, pra estudar pra prova. (N-Pred) b. Ele num voltou pra casa pra estudar pra prova não. (N-Adj)

(42) São-tomense (HAGEMEIJER, 2007, p. 232-233)

a. E na ka ba kume fa antê plaman.

(N-Pred)

3SG NEG ASP ir comer NEG antes manhã

'Até de/antes da manha, ele não comeu' 
b. E na ka ba kume antêplaman fa. (Maji ante taji). (N-Adj) 3SG NEG ASP ir comer antes manha NEG mas antes tarde 'Ele não vai comeu antes da manha (não), mas antes da tarde'

Esse efeito não decorre do número de marcadores, mas especificamente da posição pós-VP do segundo deles. Em francês, que também possui uma estrutura com dois marcadores, mas em que o segundo é imediatamente pós- $\mathrm{I}^{\mathrm{O}}$ e não pós-VP, a ambiguidade permanece (cf. (43)).

(43) a. Jean ne parle pas a cause de son ami.

J. NEG fala NEG por causa de seu amigo

b. 'É por causa de seu amigo que Jean não fala' (N-Pred)

c. 'Não é por causa de seu amigo que o Jean fala' (N-Adj)

(HAGEMEIJER; SANTOS, 2003)

Em suma, nas línguas com a estrutura [Neg-VP-Neg], o segundo marcador tem um efeito de delimitar o escopo, de marcar o limite do alcance da negação, de acordo com o seguinte esquema:
a. [Neg-VP-Neg-Adj] $\rightarrow$ apenas N-Pred
b. [Neg-VP-Adj-Neg] $\rightarrow$ apenas N-Adj

\section{Proposta}

O objetivo principal deste artigo foi apresentar os problemas existentes para a análise baseada exclusivamente em diferenças de c-comando. Em meu ponto de vista, esses fatos mostram que o escopo negativo deve ser definido pela conjugação do c-comando e alguma outra propriedade formal, ainda a ser identificada pela teoria sintática. Por limitações de espaço, poderei aqui apenas sugerir, sem desenvolver, uma proposta de análise para este fenômeno?

${ }^{9}$ Para mais detalhes, remeto o leitor a Cavalcante (2012, cap. 4, e no prelo). 
Defendo que a ambiguidade de escopo pode ser derivada pela proposta de Hornstein e Nunes (2008) sobre opcionalidade da operação de rotulação.

Para derivar as diferenças entre argumentos e adjunto, que se tornaram nebulosas no modelo de Bare Phrase Structure (CHOMSKY, 1995; remeto o leitor ao artigo de H\&N para os detalhes técnicos), os autores propõem dividir a operação de Merge em duas operações: concatenar (que conecta dois objetos sintáticos) e rotular (que torna dos dois objetos concatenados em um único objeto sintático para as demais operações da gramática). Eles propõem ainda que os argumentos são sempre concatenados e rotulados, enquanto, nos adjuntos, pode haver apenas concatenação, com a rotulação sendo opcional.

Nesta perspectiva, o processo de adjunção é menos complexo do que a introdução de um argumento (por envolver menos operações), ao contrário do que consideram as teorias tradicionais sobre adjunção. A adjunção (sem rotulação) também resultaria em uma estrutura em que há menos integração sintática entre os elementos concatenados, o que explicaria uma série de características dos adjuntos, como o fato de que um adjunto não altera o nível da categoria sintática a que se adjunge e a opcionalidade de movimento do adjunto com um predicado movido, como em (45).

(45) a. John could [eat the cake in the yard] and [eat the cake in the yard] he did.

b. John could [eat the cake in the yard] and [eat the cake] he did [in the yard ].

Em (45a), o adjunto foi concatenado ao VP e rotulado, formando um novo VP, que abrange o adjunto. Por isso, o adjunto é levado junto com o VP no processo de topicalização. Já em (45b), o adjunto estaria apenas concatenado ao VP, mas não rotulado; quando o VP é topicalizado, o adjunto, não plenamente integrado à estrutura, é deixado para trás.

Com base em H\&N, proponho que, além do c-comando, a rotulação é necessária para colocar o adjunto sob o escopo da negação. Assim, a ambiguidade de escopo negativo pode ser derivada da seguinte forma. O adjunto sempre está em uma posição abaixo de $\mathrm{I}^{\circ}$ e da negação 
(provavelmente no VP). Quando ele é concatenado e rotulado (cf. (46c)), torna-se plenamente integrado à estrutura sintática, caindo sob o escopo da negação (pré-VP) e gerando a interpretação de $\mathbf{N}$-Adj e resultando em uma curva entonacional única.

Quando o adjunto é concatenado, mas não rotulado (cf. (46b)), não se torna plenamente integrado à estrutura sintática e não cai sob o escopo da negação (pré-VP). O resultado é a leitura de N-Pred e a criação de duas curvas entonacionais.

$(46)^{10}$ a. Maria num foi promovida por ser negra.

b. Maria num foi [ ${ }_{\mathrm{vp}}$ promovida ]

por-ser-negra. (N-Pred)

c. Maria num foi $\left[{ }_{\mathrm{VP}}\left[{ }_{\mathrm{VP}} \text { promovida }\right]^{\wedge}\left[{ }_{\mathrm{PP}} \operatorname{por}^{\wedge} \operatorname{ser}^{\wedge}\right.\right.$ negra $\left.]\right]$

(N-Adj)

E quanto às sentenças com [Neg-VP-Neg]? Tais sentenças podem ser consideradas justamente a evidência em favor da análise proposta em (46), pois envolvem movimento de constituinte, levando opcionalmente o adjunto.

Assumindo que o segundo marcador é gerado em uma posição acima de CP/TP e dispara o movimento de toda a sentença para o seu especificador (cf. Cavalcante, 2007, 2012; Teixeira de Sousa, 2008 e Hansen, 2009), a posição do segundo marcador negativo em relação ao adjunto (e as leituras resultantes disso) também é resultado da opcionalidade da rotulação.

Quando há rotulação do adjunto (e a leitura N-Adj), este está totalmente integrado ao VP e pode ser movido, junto com toda a sentença/CP para o especificador da categoria em que o segundo marcador é gerado, como em (47a). Quando não há rotulação (e, portanto, há N-Pred), o adjunto não está totalmente integrado e, quando a sentença/CP é movida, o adjunto não pode ser movido junto, mas é "deixado para trás”, como em (47b).

${ }^{10}$ Nas representações, o símbolo ${ }^{\wedge}$ marca a concatenação. 
a.

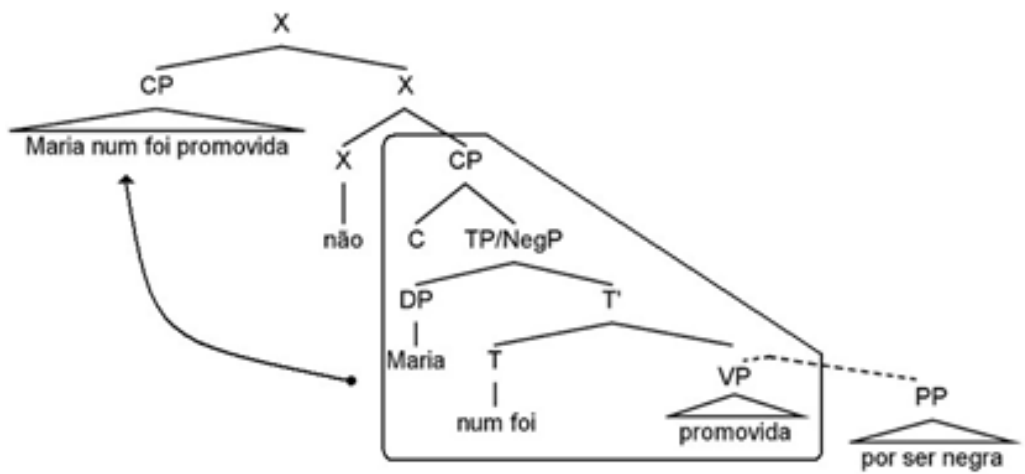

b.

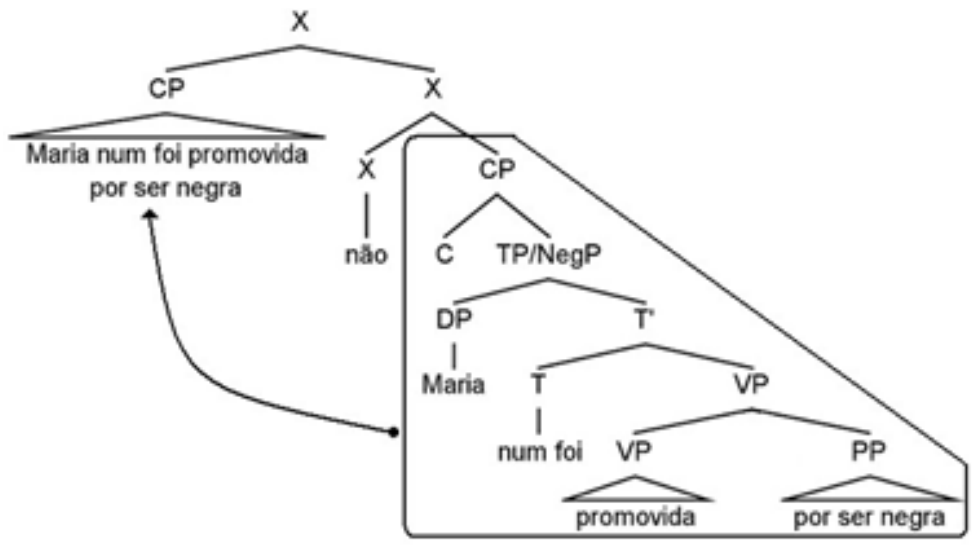

O funcionamento do marcador negativo pós-VP como um delimitador de escopo, algo problemático para a análise baseada exclusivamente em ccomando, torna-se algo previsível dentro da análise que conjuga c-comando e rotulação.

\section{Conclusões}

Discuti o fenômeno da ambiguidade de escopo negativo com adjuntos verbais e apresentei uma série de fatos que trazem problemas para as análises 
baseadas em diferentes relações de c-comando. Mostrei que, em diversos contextos, a ambiguidade permanece mesmo havendo c-comando por parte da negação sobre o adjunto. Concluí, então, que o c-comando é uma condição necessária para o escopo negativo, mas não suficiente. Apresentei um resumo breve da proposta de que os dados podem ser explicados através da adoção da ideia de Hornstein e Nunes (2008) de cisão da operação de Merge em duas operações distintas, Concatenar e Rotular, com a opcionalidade da rotulação nos casos de adjunção. Essa proposta explica a possibilidade de os marcadores negativos pós-VP antecederem ou seguirem os adjuntos causais, finais e de finalidade, bem como o fato de que a posição desse segundo marcador aparentemente marcar o limite máximo do escopo do marcador negativo pré-verbal. Os casos de controle e licenciamento de anáfora pelo sujeito, igualmente problemáticos para a teoria do c-comando, não trazem problema para a análise de rotulação, pois o c-comando é preservado em ambas as leituras.

\section{Referências}

CAVALCANTE, R. A negação pós-verbal no português brasileiro: análise descritiva e teórica de dialetos rurais de afrodescendentes. 2007. Dissertação (Mestrado em Letras) - Universidade Federal da Bahia, Salvador.

CAVALCANTE, R. Negação anafórica no português brasileiro: negação sentencial, negação anafórica e negação de constituinte. 2012. Tese (Doutorado em Linguística) - Universidade de São Paulo, São Paulo.

CHOMSKY, N. Bare Phrase Structure. In: CAMPOS, H.; KEMPCHINSKY, P. (Org.). Evolution and revolution in Linguistic Theory: Essays in honor of Carlos Otero. Washington: Georgetown University Press, 1995. p. 51-109.

CHOMSKY, N. Minimalist inquiries: the framework. In: MARTIN, R.; MICHAELS, D.; URIAGEREKA, J. (Org.). Step by step: Essays on minimalist syntax in honor of Howard Lasnik. Cambridge, MA: MIT Press, 2000. p. 81-155. 
DIECK, M. La negacion en palenquero: análisis sincrónico, estudio comparativo y consecuencias teóricas. Frankfurt: Vervuert, 2000.

FONSECA, H. D. C. Marcador negativo final no Português Brasileiro. Cadernos de Estudos Lingüisticos, Campinas, v. 46, n. 1, p. 5-19, 2004.

HAGEMEIJER, T. Clause structure in Santome. 2007. Doctoral Dissertation. University of Lisbon.

HAGEMEIJER, T.; SANTOS, A. L. Elementos polares na periferia direita: negação aparentemente descontínua, afirmação enfática e tags. In: ENCONTRO DA ASSOCIAÇÃO PORTUGUESA DE LINGÜÍSTICA, 19., 2003, Lisboa. Actas... Lisboa, 2004. p. 465-476.

HANSEN, Q. M. Negation in Brazilian Portuguese. 2009. University of Florida. (mimeo).

HORNSTEIN, N.; NUNES, J. Adjunction, labeling, and Bare Phrase Structure. Biolinguistics, v. 2, n. 1, p. 57-86, 2008.

HUANG, C.-T. J. Logical relations in Chinese and the Theory of Grammar. 1982. Doctoral Dissertation. Massachusetts Institute of Tecnology, Boston.

JOHNSTON, M. Because clauses and negative polarity licensing. In: MEETING OF THE EASTERN STATES CONFERENCE ON LINGUISTICS, 10., 1994, Ithaca (NY). Escol '93: Proceedings of... Ithaca: Cornell University Press, 1994. p. 163-174.

KAYNE, R. The antisymmetry of syntax. Cambridge: MIT, 1994.

LAKA, I. Negation in syntax: on the nature of functional categories and Projetions. 1990. Tese (Doutorado em Linguística) - Massachusetts Institute of Tecnology, Boston.

LASNIK, H. Analysis of negation in English. 1972. Doctoral Dissertation. Massachusetts Institute of Tecnology, Boston.

LASNIK, H; SAITO, M. On the subject of infinitives. Chicago Linguistic Society, Chicago, v. 27, p. 324-343, 1991. 
LINEBARGER, M. C. Negative polarity and grammatical representation. Linguistics and Philosophy, v. 10, n. 3, p. 325-387, ago. 1987.

MARTINS, A. M. Clíticos na história do português. 1994. Tese (Doutorado em Letras) - Universidade de Lisboa, Lisboa.

MARTINS, E. E. Sentential negation in spoken Brazilian Portuguese. 1997.

Dissertação (Mestrado em Linguística) - Georgetown University, Washington.

MIOTO, C. Negação sentencial no português brasileiro e a teoria da gramática. 1992. Tese (Doutorado em Linguística) - Universidade Estadual de Campinas, Campinas.

NAMIUTI, C. Negação sentencial na diacronia do português: variação com estabilidade. Revista de Estudos da Linguagem, Belo Horizonte, v. 16, n. 2, p. 193-239, 2008.

OUHALLA, J. The structure and logical form of negative sentences. Linguistic Analysis, v. 27, p. 220-244, 1997.

POLLOCK, J-Y. Verb movement, universal grammar, and the structure of IP. Linguistic Inquiry, Cambridge, v. 20, p. 365-424, 1989.

TAKUBO, Y. On the scope of negation in Japoneses. Seoul Papers in Formal Grammar Theory, 1985. Proceedings of the Third Korean-Japonese Joint Workshop, 19-21 de dezembro de 1983.

URK, C. van. Aspects of control. 2010. Dissertação (Mestrado em Linguística) - Universidade de Utrecht, Utrecht.

TEIXEIRA DE SOUSA, L. Doubled items and focus in BP: negation doubling. In: WORKSHOP INTERFACES, 2008, Campinas.

VITRAL, L. A negação: teoria da checagem e mudança lingüística. DELTA, São Paulo, v. 15, n. 1, p. 57-84, 1999.

Recebido em: 20/05/2015 Aceito: $17 / 08 / 2015$ 